\title{
Ambulation of hospitalized patients: Knowledge, values, and barriers of direct care providers
}

\author{
Melissa Thomassy, Aline "Lynn" Moore, Ashley Peacock, Justin Wright, Peggy Ward-Smith* \\ North Kansas City Hospital, United States
}

Received: October 17, 2020

Accepted: November 8, 2020

Online Published: July 30, 2020

DOI: $10.5430 / \mathrm{cns} . v 8 \mathrm{n} 4 \mathrm{p} 60$

URL: https://doi.org/10.5430/cns.v8n4p60

\begin{abstract}
The desire to ambulate hospitalized patients is tempered by their risk of falling. Research articulates the health-related benefits of ambulation, yet routinely providing this intervention is challenging. This descriptive survey-design study obtained data from consented licensed and unlicensed direct-care providers, which assessed their knowledge, values, and perceived barriers associated with routine ambulation of patients receiving care in a hospital setting. Analyses of these data conclude that the subjects were knowledgeable about and value ambulating patients. The most frequently cited barrier to routine ambulation was an inadequate staff number, followed closely by an unexpected rise in volume and patient acuity. Interventions aimed at improving the ambulation of patients should include the results of this study.
\end{abstract}

Key Words: Ambulation, Inpatient, Knowledge, Values, Barriers

\section{INTRODUCTION}

The correlation of improved patient outcomes to ambulation while receiving care in acute health settings has been welldocumented. Improved patient outcomes include shorter lengths of stay, ${ }^{[1]}$ a decreased risk for immobility-related pneumonia, ${ }^{[2]}$ and prevention of delirium. ${ }^{[3]}$ Routine ambulation has been identified as a significant variable in reducing the loss of lower extremity strength, ${ }^{[4]}$ physical activity stamina, and the ability to perform activities of daily living ${ }^{[5]}$ independently. While ambulation has documented benefits for the patient, assisting a patient to ambulate exposes the care provider to musculoskeletal injury. ${ }^{[6]}$ Additionally, when ambulating a patient with musculoskeletal weakness, there is an increased risk of them falling, which may have unintended consequences for the care provider. ${ }^{[7]}$ One of these consequences, according to Covinsky, Pierluissi, and Johnston, ${ }^{[8]}$ is "a climate of fear of falling" (p. 1,783) where nurses "feel that if somebody falls on their watch, they'll be blamed for it". ${ }^{[9]}$ (p. E1). Barriers, such as a "blame" culture, may dominate knowledge and values associated with the ambulation of patients. ${ }^{[10]}$ The purpose of this survey-design study was to assess the knowledge and values of direct care providers toward ambulation and to allow these participants to describe the barriers that inhibit the routine ambulation of hospitalized patients.

The desire to globally assess the situation resulted from performance improvement (PI) activity on the oncology unit. Najafpour and associates ${ }^{[11]}$ described the pathology of cancer and the effects of treatment places oncology patients at a higher risk for falls and an injury related to a fall. Routine outcome metrics noted an increase in falls and fall-related injuries. The oncology unit opted to participate in the Missouri Hospital Association's (MHA) rapid cycle improvement initiative "Get UP". ${ }^{[12]}$ Content experts, best practices, and PI

*Correspondence: Peggy Ward-Smith; Email: wardsmithp@umkc.edu; Address: North Kansas City Hospital, Missouri, 64116, United States. 
tools were provided by the MHA. Falls and falls with injuries were identified as outcome measures, with ambulation data documented within the activity of daily living (ADL) medical record section. After six months, the project intervention was assessed with data indicating that the incidences of falls decreased, and no fall-related injury occurred.

Before initiating the interventions hospital-wide, members of the Research and Innovation Council (RIC) determined that survey data would provide stakeholder buy-in necessary for successful adoption.

\section{REVIEW OF THE LITERATURE}

Guided by the keyword of "knowledge", "values", "ambulation barriers", "inpatient", "acute care settings", "direct care providers", "ambulation", and "survey", while limiting the review to research performed in the United States (U.S.), published with the previous 10 years in peer-review journals, and using the search engines of PubMed, Ovid, Web of Science, the Cochrane Library, and CINAHL, 75 articles were initially identified. Excluding articles which defined ambulation as missed care, assessed the decline in walking ability due to hospitalization, or evaluated an intervention retained 15 articles. These were reviewed for appropriateness, with 12 removed for (1) opinion pieces $(n=2)$, (2) data obtained from healthcare settings not reflective of this study (intensive care and rehabilitation units $[n=4]$ ), (3) duplicate publications $(n=3)$, the study population was the non-nursing staff $(\mathrm{n}=2)$, and (4) a dissertation $(\mathrm{n}=1)$. In addition to these three articles, two more were identified by searching the references of a synthesis review paper; these were then included in the review. Repeating the search and adding "barriers" as a keyword was done due to the focus difference. The results of this review identified four articles focused on personal or system barriers.

Qualitative data were obtained from eight nursing staff members for a QI project performed by Johnson and Howell. ${ }^{[13]}$ The results of the individual semi-structured interviews reveal that the staff participants articulated an understanding of the benefits associated with ambulation. The other qualitative study analyzed interview data from 25 registered nurses who provided direct care to older adults (defined as older than 65 years) on general medical/surgical units from two urban hospitals located in southern Wisconsin. ${ }^{[14]}$ Using grounded dimensional analysis techniques, a conceptual model was developed. Ambulation, as perceived by these nurses, was a means to another end and not as an end itself. All participants acknowledged that ambulating patients was the right thing to do and recognized that it was not always done. Preventing complications, specifically DVTs, pneumonia, and pressure ulcers, were identified outcomes ambulation prevented. Lack

Published by Sciedu Press of knowledge was not a significant barrier to ambulation. Suggested interventions include establishing ambulation as a standard of care, with clear measures for accountability.

Sepulveda-Pacsi and associates ${ }^{[15]}$ administered a modified version of the "Missed Nursing Care Survey" to 192 nurses who provided direct patient care from one of two different teaching hospitals in northern Manhattan. Analyses of these data identified a small association between ambulation knowledge, years of experience $(p=.04)$, and shift worked ( $p$ $=.04$ ). Regression analyses identified an inadequate number of staff (clerical and nursing) as the primary factor (80\%) preventing routine ambulation, with urgent patient situations (74.4\%) and an unexpected rise in patient volume and acuity $(67.7 \%)$ as contributory variables.

A systematic review of 38 articles, together with a metaanalysis of seven articles completed by Fazio and colleagues, ${ }^{[16]}$ included seven articles when study data were obtained within the U.S. Of these, two met the publication timeframe inclusion criteria. Prospective data from Ostir and colleagues ${ }^{[17]}$ were collected from 224 older adults by an activity monitor, with no intervention provided. Using multivariate survival models, a decline in steps from the first to the last 24 hours of a hospital stay was associated with a more than four times greater risk of death when followed for two years. Fisher and associates ${ }^{[18]}$ used accelerometers to describe ambulation activity (steps/day) among inpatients. These data describe a low-level of ambulation for all patients, with a mean of $4.1 \%$ of their day. Increased ambulation appeared to correspond to the recovery process, regardless of age, and improved physiological status as determined by vital sign stability. These authors conclude that knowledge of immobility, and its consequences, provide baseline data, serving as comparative data for interventions. The amount, duration, and timing of ambulation for inpatients, was not reported.

Previous research has identified personal and system barriers as impacting the ambulation of inpatients. Personal barriers include inadequate knowledge regarding ambulation, an inability to determine if the patient is able to ambulate safely, and the fear of professional consequences if the patient falls during the activity. ${ }^{[19]}$ System barriers, described by King and colleagues, ${ }^{[20]}$ include inadequate knowledge regarding ambulation, an inability to determine if the patient can ambulate safely, and the fear of professional consequences if the patient falls during the activity. ${ }^{[19]}$ Demanding nurse-topatient ratios; the presence of catheters, restraints, and intravenous lines; physician activity orders as bedrest; pressure on care providers to decrease or prevent falls; and inadequate interprofessional communication are additional identified 
system barriers. ${ }^{[21,22]}$

\section{ETHICS}

Institutional approval to obtain data was secured from the study site's Institutional Review Committee (IRC). Data were collected electronically using a web-based format with consent implied upon submission of survey responses.

\section{THE STUDY AND SETTING}

The clinical setting for this descriptive study was a 451 bed, not-for-profit community hospital located in the Kansas City, Missouri, metropolitan area. The hospital has a shared governance structure with RIC dedicated to enhancing scholarly knowledge and activities of nurses. In 2019, the members of the RIC became aware of the success of a PI project, completed by nurses in the acute care oncology / medicalsurgical unit, where enhanced patient mobility resulted in a sustained reduction in falls with injury. The PI project identified knowledge, values, and perceived barriers as gaps to patient ambulation in inpatient acute care. These constructs served as the framework for this study and guided the review of the literature. Survey items were developed from the literature review; these results will guide educational and policy interventions.

\section{Methods}

The study-specific survey was designed to allow participants to describe their perception of ambulation, specifically within the domains of knowledge, value, and barriers. The tool was modified from the Sepulveda-Pacsi, Soderman, and Kertesz $^{[15]}$ questionnaire to reflect organizational culture, personal experience, and policies. The study survey encompassed 35 items: 10 demographic items, three knowledge items, six value items, 15 barrier items, and one open-ended question.

Access to the survey was through a password-protected organizational email, and no identifying information was collected. Potential study participants included all registered nurses (RNs), licensed practical nurses (LPNs), and unlicensed assistive personnel (UAPs) with job responsibilities that include providing direct care more than $50 \%$ of the time. This qualifier was used to obtain access to the survey and was the only exclusion criteria.

The study email invitation was sent to the hospital's "All Nurse" and "All Nurse Tech" distribution lists by the Chief Nursing Officer. The email had an embedded consent statement and an electronic survey link. Participation in the survey was voluntary. Any item could be left blank, and participation could be terminated at any time during the participant's survey without any impact on their employment status. Within the nursing distribution list, there were 823 eligible RNs and LPNs and 214 eligible UAPs. Study data were collected for one month with a reminder email sent at the 15-day mark.

Survey data were collated by the site coordinator, checked for accuracy, and sent to a consultant for statistical analysis. Consultant electronic files were deleted after summation, and the site coordinator data remains a single-sign-on passwordprotected electronic document; no hard copies exist.

\section{RESULTS}

Of 1,037 (823 to licensed personnel [RN and LPN] and 214 to UAPs) study invitations sent, 179 individuals accessed the survey, with 171 meeting study inclusion criteria. Eight individuals accessed the study survey and were prevented from participation because their job responsibilities did not include direct patient care. This finding represents a $16.48 \%$ response rate $(171 / 1,037)$. Separating the responses by job classification reveals 107 responses from licensed personnel (RNs) and 64 responses from those in various UAP roles, with no responses from LPNs. Thus, the response rate among RNs was $13 \%$ (107/823), with a $29.90 \%$ response rate from UAPs (64/214). While each response rate is acceptable, the difference in participation based on job classification reveals the topic to be of interest for UAPs, which resulted in the development of study sub-populations (licensed and non-licensed personnel). Study results are described as percentages to compensate for the numerical differences in each survey's possible responses and sample size of the subpopulations. Thus, all study results are presented by the entire study population, then based on the responses of each study sub-population (licensed and unlicensed personnel).

\subsection{Demographic results}

Demographically, participants in this study were primarily female $(93.1 \%), 61.5 \%$ were under the age of 41 years, employed full-time $(66.5 \%)$, working day shifts $(66.4 \%)$, at their present position for five years or less $(51 \%)$, with a bachelor's degree $(47.5 \%)$. When comparing the study sub-populations, $55.1 \%$ of the RNs, while $78.7 \%$ of the UAPs were under 41 years. Unlicensed participants were less likely to be working full-time, more likely working on a night shift, and employed in their present position less than six years $(68.2 \%$ versus $43 \%$ ). A higher level of education was described by the RNs, with $69.2 \%$ reporting a bachelor's degree, a level of education reported by $10.9 \%$ of the UAPs. All participants were aware that ambulation is a job responsibility of their current position. 


\subsection{Knowledge results}

All participants were aware of safe ambulation practices, the health outcome effects when ambulation is a part of the plan of care, and how to determine readiness for safe ambulation. Comparing sub-population responses reveals similar responses, except safe patient ambulation practices. Yet, the UAPs articulate extreme knowledge concerning safe ambulation practices (82.2\% versus 6.6\%). Knowledge items results are displayed in Table 1.

Table 1. Knowledge item results

\begin{tabular}{|c|c|c|c|c|}
\hline Knowledge Item & Possible Response & Entire Population & Licensed Personnel & Unlicensed Personnel \\
\hline \multirow{3}{*}{$\begin{array}{l}\text { I am knowledgeable about safe patient ambulation } \\
\text { practices. }\end{array}$} & Somewhat & $4.2 \%$ & $5.8 \%$ & $1.6 \%$ \\
\hline & Very & $27.1 \%$ & $33.6 \%$ & $16.1 \%$ \\
\hline & Extremely & $68.7 \%$ & $60.6 \%$ & $82.3 \%$ \\
\hline \multirow{3}{*}{$\begin{array}{l}\text { I am knowledgeable about the effect patient } \\
\text { mobility/immobility has on patient health } \\
\text { outcomes. }\end{array}$} & Somewhat & $3 \%$ & $2.9 \%$ & $3.3 \%$ \\
\hline & Very & $26 \%$ & $26.9 \%$ & $24.6 \%$ \\
\hline & Extremely & $71 \%$ & $70.2 \%$ & $72.1 \%$ \\
\hline \multirow{3}{*}{$\begin{array}{l}\text { I am knowledgeable about how to determine a } \\
\text { patient's readiness for safe ambulation. }\end{array}$} & Somewhat & $13.3 \%$ & $12.5 \%$ & $14.7 \%$ \\
\hline & Very & $38.2 \%$ & $39.4 \%$ & $36.1 \%$ \\
\hline & Extremely & $48.5 \%$ & $48.1 \%$ & $49.2 \%$ \\
\hline
\end{tabular}

\subsection{Value results}

Responses to the six items which allowed each participant to describe their perception of the value of ambulation had notable differences. Responses from UAPs describe a greater perception that patient ambulation is a part of their job re- sponsibilities, something they include in their patient instruction, and improves patient quality of life. All participants displayed a value perception that supports ambulation as a workload item and should be included in the daily care plan. The value item results are displayed in Table 2.

Table 2. Value perception item results

\begin{tabular}{|c|c|c|c|c|}
\hline Value Perception Item & $\begin{array}{c}\text { Possible } \\
\text { Response }\end{array}$ & $\begin{array}{c}\text { Entire } \\
\text { Population } \\
\end{array}$ & $\begin{array}{c}\text { Licensed } \\
\text { Personnel } \\
\end{array}$ & $\begin{array}{c}\text { Non-licensed } \\
\text { Personnel }\end{array}$ \\
\hline \multirow{3}{*}{ Patient ambulation, as a workflow item, is ... } & Neutral & $4.8 \%$ & $6.4 \%$ & $1.9 \%$ \\
\hline & Important & $42.8 \%$ & $40.9 \%$ & $46.2 \%$ \\
\hline & Very Important & $52.4 \%$ & $52.7 \%$ & $51.9 \%$ \\
\hline \multirow{3}{*}{ Patient ambulation, as one of my job responsibilities, is } & Neutral & $7.7 \%$ & $10.9 \%$ & $1.9 \%$ \\
\hline & Important & $45.1 \%$ & $47.8 \%$ & $40.4 \%$ \\
\hline & Very Important & $47.2 \%$ & $41.3 \%$ & $57.7 \%$ \\
\hline \multirow{3}{*}{$\begin{array}{l}\text { When doing patient education, including the benefits } \\
\text { of ambulation is ... }\end{array}$} & Neutral & $4.8 \%$ & $5.3 \%$ & $3.9 \%$ \\
\hline & Important & $35.9 \%$ & $39.8 \%$ & $28.8 \%$ \\
\hline & Very Important & $59.3 \%$ & $54.9 \%$ & $67.3 \%$ \\
\hline \multirow{3}{*}{$\begin{array}{l}\text { Ambulation, as an intervention to prevent } \\
\text { complications while hospitalized is .... }\end{array}$} & Neutral & $2 \%$ & $3.2 \%$ & $0 \%$ \\
\hline & Important & $28 \%$ & $27.7 \%$ & $28.9 \%$ \\
\hline & Very Important & $70 \%$ & $69.1 \%$ & $71.1 \%$ \\
\hline \multirow{3}{*}{$\begin{array}{l}\text { Among interventions aimed at improving quality of } \\
\text { life, ambulation is ... }\end{array}$} & Neutral & $3.4 \%$ & $5.3 \%$ & $0 \%$ \\
\hline & Important & $32.9 \%$ & $34 \%$ & $28.8 \%$ \\
\hline & Very Important & $63.7 \%$ & $60.7 \%$ & $69.2 \%$ \\
\hline \multirow{3}{*}{$\begin{array}{l}\text { Including ambulation in a patient's daily plan of care is } \\
\ldots\end{array}$} & Neutral & $2.8 \%$ & $3.2 \%$ & $1.9 \%$ \\
\hline & Important & $33.8 \%$ & $34.4 \%$ & $32.7 \%$ \\
\hline & Very Important & $63.4 \%$ & $62.4 \%$ & $65.4 \%$ \\
\hline
\end{tabular}


Table 3. Barrier item results

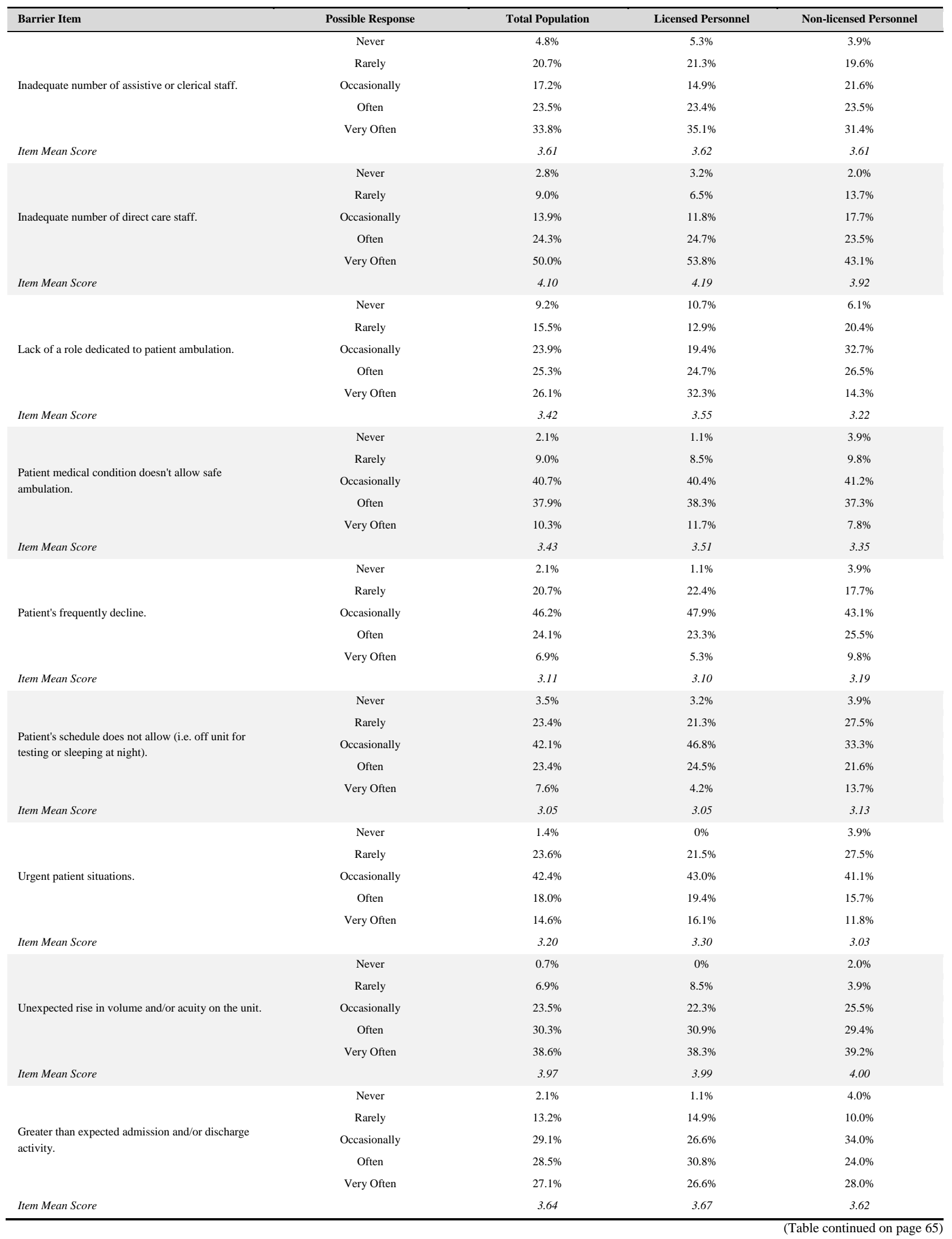


Table 3 (continued.)

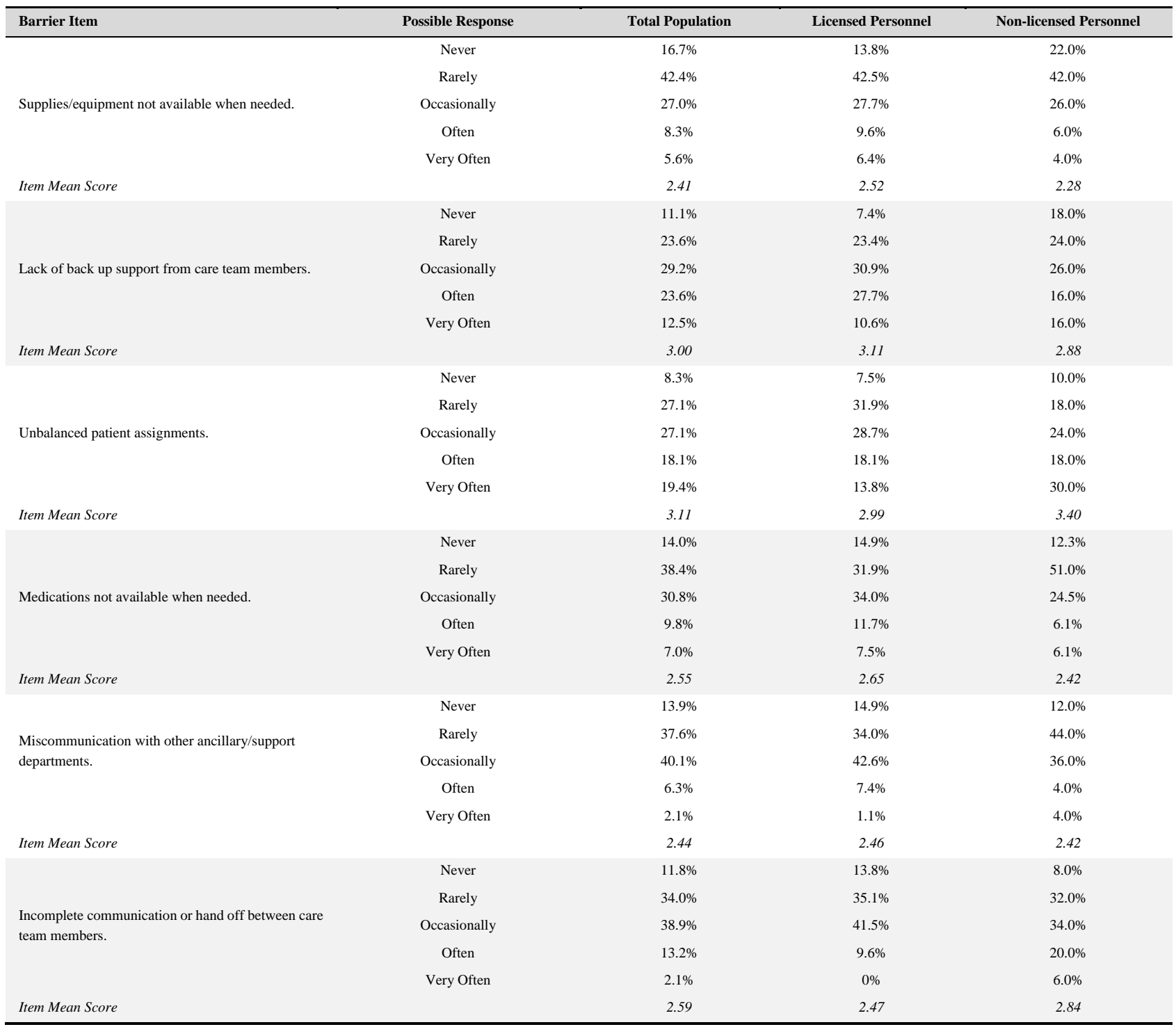

\subsection{Barrier results}

Barrier results were initially analyzed by percent responses. This provides a view of the opinion of the participants. Each response was then ranked from 1 (neutral) to 5 (very often). Transferring the responses into ordinal data allowed means to be calculated. Scores higher than 3.5 would reflect the barrier, perceived by these participants, that occurs at least $70 \%$ of time. Thus, a mean cutoff score of 3.5 was used to identify a significant barrier.

Guided by mean scores, an inadequate number of direct care staff (4.10), followed closely by an unexpected rise in volume and acuity of the unit (3.97), were the most frequently cited barriers to patient ambulation. These items also attained the highest mean within each sub-population, meaning the experience was universal. Other high-scoring items include greater than expected admission and discharge activity (3.64) and an inadequate number of assistive or clerical staff (3.61). Supplies/equipment not available when needed (2.41) and miscommunication with other ancillary/support departments (2.44) achieved the lowest mean scores, indicating that they infrequently impact patient ambulation activity.

Certainly, responses that attained mean scores above the $50 \%$ rank should be reviewed; of the 15 barrier items, 12 (80\%) achieved a median score of 2.5. By reporting on the items at the extreme end of the analyses, we wish to identify the greatest and least important items. Barrier item results are displayed in Table 3.

\subsection{Open-ended item responses results}

The final item on the survey was open-ended and allowed each participant to describe their feelings associated with 
patient ambulation. The open-ended statement started as "When I can ambulate patients, I feel ..." There were 119 responses submitted. These data were analyzed using content analysis techniques, guided by HyperRESEARCH. ${ }^{[23]}$ Once significant statements were identified, they were placed into positive and negative categories. The majority $(65 \%)$ of these responses described positive feelings associated with the ability to ambulate a patient. Theme development was guided by terms used by the participants and classified as benefits (positive) or frustrations (negative) feelings.

Benefit exemplars completed the open-ended statement as: When I am able to ambulate patients, I feel ...

- that I am helping them get one step closer to their discharge goals

- Accomplished... like I'm going above and beyond because I actually have the time to. I am aware of the importance but am often stretched too thin.

- like I have helped improve their hospital stay, their mood occasionally, and their physical health partially. I understand the importance of mobility practices while in the hospital due to the need for bed alarms/chair alarms at all times.

- sometimes like I have other things to do and wish there were someone who could do these simple but important tasks. other times its nice to have that moment with the patient to converse and assess them at the same time.

- I enjoy being able to ambulate my patients. It gives us time to discuss things. I know it helps build their strength and the change of scenery and exercise is good for them mentally and emotionally. Nurses don't just heal patients with medication. Ambulating is vital but unfortunately, so time consuming it becomes a low priority.

Frustration exemplars completed the open-ended statement as: When I am able to ambulate patients, I feel ...

- like I'm completing an extra special task instead of something that should feel like a routine part of patient care.

- That I have 10 million other things I need to do at the same time.

- Nervous, ... I am acutely aware that I am now in an impossible situation where I am responsible for two patients labeled a fall risk ... I am aware that an adequate amount of support staff is provided to prevent falls and encourage ambulation.

- When we are staffed and not overly busy I feel happy to take the time to walk and glad to talk with them. When we are short-staffed, it is difficult to find the time, but if we can find the time, it often feels like a chore because, at a certain point, we need a break to sit down and rest our feet during the 12 hours.

\section{RESUlts}

Data from this study describe a nursing staff, both licensed and unlicensed, that is knowledgeable about the benefits of ambulation and how to assess for ambulation readiness. A greater number of UAPs perceive ambulation as a part of their job responsibility and aware that this information should be included in patient instruction. Responses from UAPs also describe the value perception that ambulation improves their quality of life. These results mirror previous research ${ }^{[15]}$ yet vary from the findings of Hughes. ${ }^{[19]}$ With the exception of the availability of supplies and equipment, each barrier item negatively impacted the ability to ambulate patients by $50 \%$ of the total study population. Thus, despite the knowledge and a positive valuing of ambulation, there are many reasons why this activity is not routinely implemented.

Limitations associated with this study include the use of a single study site, data collection using a web-based format, and a limited data collection interval. The use of a study and site-specific survey, despite the use of research evidence to guide item identification, limits the generalizability of these results. There may be variables other than knowledge, value, and perceived barriers that inhibit ambulating patients in an inpatient setting. Certainly, replication of this study, with the addition of items of interest to the study site, should occur before developing interventions to increase the ability to ambulate patients receiving care in a hospital setting.

\section{Discussion}

Routine ambulation of inpatients, while perceived as important, is not routinely accomplished. The most-reported barriers were inadequate staffing and an unexpected rise in volume and acuity of the unit. An inadequate number of staff may not become apparent until admissions or care acuity rises. Thus, while flexible staffing may address this barrier, the ability to predict, and alter the staffing, is not possible. Acknowledging that staffing any inpatient unit appropriately is challenging and may change during one specific shift does impact the ability to ambulate patients. Mechanical interventions, such as an Ambulatory Platform Apparatus, ${ }^{\text {[24] }}$ activity monitors, ${ }^{[17]}$ and Accelerometers, ${ }^{[24]}$ have been recommended as ambulation assistive devices/monitors. A more flexible intervention, providing a staff member dedicated to ambulation activities, assessed in a randomized control trial by Hamilton and associates, ${ }^{[26]}$ improved mobility while decreasing hospital length of stay for patients in the intervention group. 
Workflow and the care delivery model at the study site may have influenced responses to both knowledge and value perceptions. As ambulation, especially to and from toileting, is considered a component of ADLs, licensed nurses are routinely delegated to a UAP. Since ADLs and ambulation are often delegated to a UAP, the study site has historically provided more training and emphasis on these care items. The oncology unit at the study site that conducted the PI around increasing ambulation additionally instituted a dedicated UAP for ambulation, which may have influenced the PI results.

Responses to the open-ended question describe positive professional-related feelings and the opportunity to engage and teach patients while ambulating them. These data also describe ambulation as "low" on the list of patient care activities. The personal and professional repercussions related to a patient fall should be minimized. While the study site embraces a non-punitive environment, reporting the fall to family and healthcare providers, along with completing the required documentation, is time-consuming and personally stressful. While Hughes ${ }^{[19]}$ describes professional consequences related to patient falls, and these have been relegated to being non-punitive, the phenomenon may still be present. These participants described feeling extremely knowledgeable about assessing a patient's readiness for ambulation; we did not query if a risk for falls guided the decision to ambulate; this may be an area of future research.

\section{Conclusions}

The results of this study document that direct care providers are aware that assisting patients to ambulate while receiving care in an acute care setting has health outcome benefits and is a valued activity. Despite this knowledge, and contrary to the recommendations from the $\mathrm{MHA}^{[12]}$ and the Centers for Medicare and Medicaid Services (CMS), ${ }^{[7]}$ the ambulation of patients in acute care settings is anything but routine. An inadequate number of nursing personnel and variations in the patient population's care needs are the principal barriers to including this activity in a plan of care. A greater than expected admission and discharge activity, an inadequate number of assistive or clerical staff also impact the ability to routinely ambulate inpatients. These variables represent a busy inpatient unit - a typical scenario. While additional research is always warranted, the ability to generalize these results should be considered. Providing a dedicated staff member for ambulation would bypass staffing variance barriers and change patient census and acuity levels during any shift. The literature, and these data, appear to support that intervention, despite the inherent financial risks associated with a position limited in scope. Reviewing the care needs of the patients, and the available resources, should be performed before dedicating a staff member to this task. Data should be collected to determine the efficacy of a dedicated staff member. This research will determine its impact, feasibility, and sustainability.

\section{Conflicts of InTEREST Disclosure}

The authors declare they have no conflicts of interest.

\section{REFERENCES}

[1] Dewitt K, Coto JA, Carr L, et al. Ambulation programs: Decreasing length of stay and improving outcomes. MEDSURG Nursing. 2019; 28(5): 293-296.

[2] Tesoro M, Peyser DI, Villarente F. A retrospective study of nonventilator associated hospital acquired pneumonia incidence and missed opportunities for nursing care. JONA: The Journal of Nursing Administration. 2018; 48(5): 285-291. PMid: 29672375. https: //doi.org/10.1097/NNA.0000000000000614

[3] Mudge AM, Banks MD, Barnett AG, et al. CHERISH (collaboration for hospitalised elders reducing the impact of stays in hospital): protocol for a multi-site improvement program to reduce geriatric syndromes in older inpatients. BMC Geriatrics. 2017; 17(1): 11. PMid: 28068906. https://doi.org/10.1186/s12877-016-0399-7

[4] Van Ancum JM, Pijnappels M, Jonkman NH, et al. Muscle mass and muscle strength are associated with pre- and post-hospitalization falls in older male inpatients: a longitudinal cohort study. BMC Geriatrics. 2018; 18: 116. PMid: 29769029. https://doi.org/10.1186/s1 2877-018-0812-5

[5] Valenzuela PL, Morales JS, Castillo-Garcia A, et al. Effects of exercise interventions on the functional status of acutely hospitalised older adults: A systematic review and meta-analysis. Ageing Research Reviews. 2020; 61: 1658-1637. PMid: 32330558. https://doi.org/10.1016/j.arr.2020.101076

[6] Dressner MA, Kissinger SP. Occupational injuries and illnesses among registered nurses. Monthly Labor Review, U.S. Bureau of Labor Statistics; November 2018. https://doi .org/10.21916 /mlr.2018.27

[7] Fehlberg EA, Lucero RJ, Weaver MT, et al. Impact of the CMS NoPay Policy on Hospital-Acquired Fall Prevention Related Practice Patterns. Innovation in Aging. 2017; 1(3): igx036. PMid: 29911187. https://doi.org/10.1093/geroni/igx036

[8] Covinsky KE, Pierluissi E, Johnston CB. Hospitalization-associated disability: "She was probably able to ambulate, but I'm not sure". Journal of the American Medical Association. 2011; 306: 17821793. PMid: 22028354. https://doi.org/10.1001/jama. 201 1.1556

[9] Bailey M.' Fear Of Falling': How Hospitals Do Even More Harm By Keeping Patients In Bed. Washington Post. October 17, 2019. Available from: www. washingtonpost.com/health/overzeal 
ous-in

[10] Paley DG, Corbett N. Balancing the risk. Clinical Nurse Specialist. 2016; 30(5): 250-252. PMid: 27509558. https ://doi.org/10.1 $097 /$ NUR . 0000000000000230

[11] Najafpour Z, Godarzi Z, Arab M, et al. Risk factors for falls in hospitals inpatients: A prospective nested case control study. International Journal of Health Policy and Management. 2019; 8(5): 300. PMid: 31204446. https://doi.org/10.15171/ijhpm.2019.11

[12] Missouri Hospital Association. (n.d.). 'Get UP'. PMid: 28068906. https://doi.org/10.1186/s12877-016-0399-7

[13] Johnson A, Howell DM. Mobility bridges a gap in care: Findings from an early mobilisation quality improvement project in acute care. Journal of Clinical Nursing. 2019; 28: 21-22. PMid: 31264747. https://doi.org/10.1111/jocn. 14986

[14] Doherty-King B, Bowers B. How nurses decide to ambulate hospitalized older adults: development of a conceptual framework. The Gerontologist. 2011; 51(6): 786-797. PMid: 22024979. https : //doi.org/10.1093/geront/gnr044

[15] Sepulveda-Pacsi AL, Soderman M, Kertesz L. Nurses' perceptions of their knowledge and barriers to ambulating hospitalized patients in acute settings. Applied Nursing Research. 2016; 32: 117-121. PMid: 27969013. https://doi.org/10.1016/j.apnr.2016.06.001

[16] Fazio S, Stocking J, Kuhn B, et al. How much do hospitalized adults move? A systematic review and meta-analysis. Applied Nursing Research. 2020; 51: 151189. PMid: 31672262. https: //doi.org/10.1016/j.apnr.2019.151189

[17] Ostir GV, Berges IM, Kuo YF, et al. Mobility activity and its value as a prognostic indicator of survival in hospitalized older adults. Journal of the American Geriatric Society. 2013; 61(4): 551-557. PMid: 23527951. https://doi.org/10.1111/jgs. 12170

[18] Fisher SR, Goodwin JS, Protas EJ, et al. Ambulatory activity of older adults hospitalized with acute medical illness. Journal of the American Geriatrics Society. 2011; 59(1): 91-95. PMid: 21158744. https ://doi.org/10.1111/j.1532-5415.2010.03202.x
[19] Hughes RG. Nurses at the "Sharp End" of Patient Care. In: Hughes RG, editor. Patient Safety and Quality: An Evidence-Based Handbook for Nurses. Agency for Healthcare Research and Quality (US) 2008. Chapter 2. Available from: https://www.ncbi.nlm.nih.g ov/books/NBK2672/

[20] King B, Pecanac K, Krupp A, et al. Impact of falls prevention on nurses and care of fall risk patients. The Gerontologist. 2018; 58(2): 331-340. PMid: 28011591. https ://doi.org/10.1093/geront /gnw156

[21] Jones KJ, Skinner A, Venema D, et al. Evaluating the use of multiteam systems to manage the complexity of inpatient falls in rural hospitals. Health Services Research. 2019; 54(5): 994-1006. PMid: 31215029. https://doi .org/10.1111/1475-6773.13186

[22] Lopez-Jeng C, Eberth SD. Improving hospital safety culture for falls prevention through interdisciplinary health education. Health Promotion Practice. 2019; 524839929840337. PMid: 30966813. https://doi .org/10.1177/1524839919840337

[23] Researchware. HyperRESEARCH 3.7.3. 2015. Computer Software, Researchware, Inc. Available from: http://www.researchware . com/

[24] Henecke L, Hessler K, Lalonde T. Inpatient ambulation: use of an ambulation platform apparatus. JONA: Journal of Nursing Administration. 2015; 45(6): 339-344. PMid: 26010284. https: //doi.org/10.1097/NNA.0000000000000209

[25] McCullagh R, Darren D, Horgan F, Timmons, S. Factors associated with walking in older medical inpatients. Archives of Rehabilitation and Clinical Translation. 2020; 2(1): 1-8. https ://doi .org/10.1 $016 / j$. arrct. 2020.100038

[26] Hamilton AC, Stilphen M, Hu B, et al. Dedicated ambulator-assisted physical activity to improve hospital outcomes measures in elderly medical inpatients: A randomized controlled trial. Abstract published at Hospital Medicine. 2018; April 8-11. Abstract 124. Available from: https://shmabstracts.org/abstract/dedicated-a mbulator-assisted-physical-activity-to-improve-hos pital-outcome-measures-in-elderly-medical-inpatie nts-a-randomized-controlled-trial/ 